\title{
Interações, práticas, políticas e modos de gestão: o referencial histórico cultural nas teses e dissertações do grupo INFOC de 2003 a 2013

\author{
Alexandra Pena, ${ }^{\star}$ Leonor Pio Borges de Toledo, Silvia Néli Barbosa \\ Pontificia Universidade Católica do Rio de Janeiro, Rio de Janeiro, RJ, Brasil
}

\begin{abstract}
Resumo
Tendo em perspectiva que o pesquisador que pretende investigar as crianças como sujeitos deve levar em conta quem são as crianças, como participam do processo de produção dos conhecimentos, que significados produzem sobre a realidade que as cerca, este artigo faz o levantamento da presença de Vigotski na produção acadêmica do grupo INFOC - Infância Formação e cultura, envolvendo pesquisas no periodo de 2003 a 2013. De 30 pesquisas, oito trazem Vigotski como referência para analisar interações e práticas entre crianças e destas com os adultos em creches, pré-escolas. Um estudo se realiza em um museu e dois abordam politicas de educação infantil e formação de profissionais. Este artigo faz também o levantamento dos livros utilizados como fonte de pesquisa, bem como que conceitos estão presentes. Com este levantamento, pretendeu-se verificar como o enraizamento das ideias de Vigotski, enquanto um dos autores de referência desse grupo, se ramifica nas pesquisas individuais.
\end{abstract}

Palavras-chave: Vigotski; produção acadêmica; pesquisa.

\section{Interactions, practices, policies and methods of management: the benchmark in cultural history of theses and dissertations in INFOC group 2003-2013}

\begin{abstract}
The researcher who intends to investigate children as subjects must consider who they are, how do they participate of the knowledge production process and which meanings do they produce about reality. In this perspective, this paper presents a survey of Vigotski's appearance in the academic production of INFOC - Infância, Formação e Cultura, between 2003 and 2013. Considering thirty thesis and dissertations, in eight we found Vigotski as a reference to analize interactions and practices between children and between children and adults in kindergartens and pre-schools. One study takes place in a museum and two are about policies for Early Education and teacher's training. This paper also analizes which of Vigotski's books and concepts were taken as theorical sources. This survey focused on how the ideas of Vigotski, as an reference author of INFOC group, is present in each of the individuals researches.
\end{abstract}

Keywords: Vigotski; scientific publication; research

O INFOC ${ }^{1}$ é um grupo de pesquisa sobre INfância, FOrmação e Cultura que atua desde 1993 no Departamento de Educação da PUC-Rio e é composto por professores e alunos de graduação, especialização, mestrado e doutorado. As bases teóricas para o estudo da infância, das crianças e da educação têm sido formuladas a partir de uma perspectiva interdisciplinar que tem seus fundamentos na psicologia, na sociologia e na filosofia, em especial em Mikhail Bakhtin, Lev Vigotski, Walter Benjamin e Martin Buber. O estudo desses teóricos e as contribuições da antropologia e da sociologia da infância favorecem um conhecimento denso, sensível e ético para compreender crianças e adultos e suas interações no mundo contemporâneo.

$\mathrm{Na}$ sua trajetória, o grupo vem pesquisando políticas, formação e práticas, ora centrando na dimensão macro, com o projeto realizado de 1999 a 2005, "Formação de Profissionais de educação infantil no Estado do Rio de Janeiro: concepções, políticas e modos de implementação" (KRAMER, 2001, 2005); ora na dimensão micro, com o projeto feito de 2005 a 2008, "Crianças e adultos em diferentes contextos: a infância, a cultura contemporânea e

\footnotetext{
^Endereço para correspondência: Pontifícia Universidade Católica do Rio de Janeiro PUC-Rio. Rua Marquês de São Vicente, 225 - Prédio Cardeal Leme - $10^{\circ}$ andar - sala 1049. Gávea. CEP: 22451-900 - Rio de Janeiro, RJ - Brasil. E-mail: alexandracpena@ yahoo.com.br, leonorptoledo@gmail.com, silvianeli@yahoo.com.br.

Grupo de pesquisa sobre infância, formação e cultura (INFOC), coordenado por Sonia Kramer (Pontifícia Universidade Católica do Rio de Janeiro/PUC-Rio), Maria Fernanda Rezende Nunes (UNIRIO) e Patrícia Corsino (UFRJ).
}

a educação" (KRAMER, 2009); ora em ambas as dimensões, com o projeto de 2009 a 2011, "Infância e educação infantil no estado do Rio de Janeiro: concepções e ações" (NUNES; CORSINO; KRAMER, 2013), que fez o balanço da educação infantil no estado do Rio de Janeiro, analisando políticas (1999/2009) e instituições.

Em continuidade com essa trajetória, a pesquisa em curso, intitulada "Estudos comparativos de interações, práticas e modos de gestão em creches, pré-escolas e escolas"2 focaliza interações e práticas de crianças e adultos no sentido de contribuir para enfrentar desafios relativos à qualidade das práticas de educação infantil, considerando as políticas públicas de infância e formação. O objetivo é analisar e compreender interações, práticas e modos de gestão de instituições de educação infantil para estabelecer relações, problematizar, identificar avanços e problemas nas políticas públicas e suas formas de implementação.

Uma das estratégias metodológicas da pesquisa é revisitar o campo das 30 teses e dissertações (Anexo 1) feitas, desde 2003, em creches, pré-escolas, escolas, sendo três pesquisas em espaços não escolares. Este artigo faz o levantamento da presença de Vigotski na produção acadêmica acima referida. Em quantos estudos Vigotski aparece entre os autores de referência? Que livros foram utilizados como fonte? Que conceitos estão presentes? Com este levantamento pretendeu-se verificar como o

\footnotetext{
${ }^{2}$ Todos os projetos realizados com apoio do CNPq e da FAPERJ.
} 
enraizamento das ideias de Vigotski, enquanto um dos autores de referência do grupo INFOC, se ramifica nas pesquisas individuais.

\section{Vigotski e a abordagem histórico-cultural como referencial teórico-metodológico}

Entre 18 dissertações e 12 teses feitas desde 2003, Vigotski aparece como autor de referência em 11 pesquisas. Entre essas pesquisas, oito têm como tema as práticas e interações entre as crianças e entre crianças e adultos nas creches e pré-escolas, uma aborda as interações entre crianças, jovens, adultos e obras de arte no espaço do museu e duas se referem a políticas de educação infantil e formação de profissionais. Nesses trabalhos, a abordagem histórico-cultural de Vigotski aparece como referência teórica, para análise do campo, mas também como referencial para sustentar a metodologia. Desse modo, as pesquisas do INFOC nesses dez anos estabelecem diferentes diálogos com a produção vigotskiana. Anterior a essas produções que têm como foco as interações entre crianças e destas com os adultos, bem como as políticas de formação, há um grupo de pesquisas sobre formação, leitura e escrita que não estão relacionadas neste texto (Anexo 2).

A seguir, então, apresentamos os resumos das 12 pesquisas que integram as produções de teses e dissertações desde 2003.

Corsino (2003) teve como preocupação central conhecer as concepções de infância, linguagem e letramento que permeiam os discursos e as práticas das diferentes instâncias da educação infantil da rede municipal da cidade do Rio de Janeiro. Fundamenta-se teoricamente na produção relativa à história e política da educação da criança de zero a seis anos, na concepção de criança como produtora de cultura, cidadã de direitos e na linguagem enquanto espaço das inter-relações sociais e lugar de constituição da consciência, desenvolvimento e formação. Esses fundamentos foram delineados a partir de referencial bibliográfico específico de cada eixo de estudo, mas teve como pilares as ideias de Mikhail Bakhtin, Walter Benjamin e Lev Vigotski.

Barbosa (2004) estudou interações entre crianças e adultos no cotidiano de uma pré-escola pública de educação infantil no município do Rio de Janeiro, situada no centro de um dos maiores parques florestais da cidade. A compreensão desse cotidiano leva em consideração o ponto de vista da criança, de como a criança se apropria do espaço, o que ela faz, o que diz, entendendo a importância da brincadeira para a criança conhecer o mundo e reconhecer-se no mundo. Após tratar de educação infantil, infância e criança, a autora descreve escolhas, acertos e desacertos nos quais se estrutura a pesquisa, analisa o cotidiano da educação infantil: observa interações entre crianças e adultos e das crianças com seus pares, procurando a olhar a infância a partir do que é específico das crianças - seu poder de imaginação, fantasia, criação - entendendo-as como produtoras de cultura e que nela são produzidas.
Drago (2005) investigou a inclusão de crianças deficientes nas salas regulares da educação infantil, à luz das representações sociointerativas da criança deficiente com o meio que a cerca, levando em consideração as concepções de educação, infância e inclusão. A pesquisa foi realizada em um centro municipal de educação infantil de Vitória/ES, tendo como sujeitos crianças de pré-escola com criança deficiente em processo de inclusão, a professora da turma, o corpo técnico-administrativo, o serviço de assessoramento de educação infantil e educação especial da Secretaria de Educação. Teve como pressuposto a inclusão na educação infantil do município de Vitória e buscou entender as concepções de infância e educação especial que fazem parte do discurso de quem está à frente do processo educacional (professor, diretor, pedagogo, assessor) e que se reflete na prática, no cotidiano das crianças em interação com crianças com e sem deficiência e com adultos. A pesquisa evidenciou a distância entre o que se fala acerca da inclusão e o cotidiano dos atores do processo educacional: diferentes ações no contexto pesquisado ao invés de incluir parecem excluir a partir da educação infantil.

Moura (2005) pesquisou a relação das escolas com os museus de arte, ferramenta fundamental para a formação cultural e artística das crianças. Tem origem numa pesquisa realizada em um museu de arte, focando as crianças, os jovens e os professores que aderiram, em visitas escolares, ao programa educativo, e os profissionais responsáveis por esse atendimento. A partir da observação direta de registros escritos e de imagens, a pesquisa buscou conhecer e compreender interações entre crianças, jovens, adultos e obras de arte no espaço do museu e refletir sobre essas interações no que se referem ao desenvolvimento da sensibilidade estética e da capacidade de apreciação crítica. Reconheceu a infância como construção social, compreendeu crianças e jovens como atores sociais e procurou na sociologia da Infância, na filosofia antropológica de Walter Benjamin e na psicologia de Vigotsky, as bases de interpretação.

Motta (2007) focalizou práticas de autoridade exercidas por adultos, pais e professores, sobre crianças em uma escola privada de educação infantil no Rio de Janeiro, concebendo a criança como sujeito. A compreensão dessas práticas considera o ponto de vista das crianças, o como se apropriam delas e as apresentam, entendendo a importância da brincadeira para as crianças conhecerem o mundo e, a partir da relação com seus pares e com os adultos, produzirem suas culturas a partir da cultura mais ampla onde estão inseridas. Após situar a discussão na contemporaneidade e os debates que a cercam, apresentou a escola e a família como construções históricas e nos seus aspectos concretos das instituições pesquisadas, analisando práticas de autoridade a partir da observação das interações entre crianças e adultos e das crianças entre si.

Mello (2008) buscou compreender os significados de ser professor mediador na educação de crianças pequenas. Buscou conhecer tipos de mediação realizadas por adultos e crianças de duas turmas de educação infantil em uma escola de ensino fundamental e compreender como eles se concebem e concebem suas ações na escola. 
No trabalho de campo, as relações entre crianças, adultos e signos permitiram perceber recorrências e diferenças nos tipos de mediações que ocorrem no cotidiano de cada grupo. Quanto às mediações dos adultos com as crianças das turmas de crianças de quatro anos e de crianças de cinco anos, há mediações organizadoras, desafiadoras quanto à convivência com os colegas, mediações informativas e instrutivas ("siga o modelo do professor"). No caso das mediações de crianças com crianças e com adultos, as crianças solicitam a participação direta do adulto para resolver o que sozinhas não conseguem, as que se inspiram nas falas ou atitudes do adulto sem a presença direta deste em suas ações e as mediações que vão além do modelo adulto. Nessas interações, signos revelam ideias e concepções importantes para compreender práticas escolares para/com as crianças pequenas.

Medeiros (2009) procurou identificar e compreender concepções de infância numa instituição situada na região Norte, em meio à complexidade de ações nas áreas de cultura, lazer, saúde e educação. Fez observações de interações entre adultos (das áreas de saúde, lazer, cultura e educação) e crianças (da educação infantil). Após apresentar os caminhos da pesquisa, a concepção de infância que balizou o trabalho e a instituição pesquisada, tratou de concepções de infância, espaços e organização, a ação e olhar dos adultos sobre as crianças, além de suas próprias infâncias. Por fim, as crianças ganharam lugar e voz para dizer o que é ser criança e ser adulto. Ao identificar diferentes concepções de infância e evidenciar infâncias dos adultos e seus olhares sobre as crianças, apontou a necessidade de discutir a infância na instituição e aspectos que contribuam para a reflexão de práticas de adultos que trabalham com a educação infantil.

Motta (2010) focalizou as transformações sociais na passagem da educação infantil para o ensino fundamental e a ação da cultura escolar sobre as culturas infantis, transformando os agentes sociais crianças em agentes sociais alunos. A pesquisa foi feita em uma escola do município de Três Rios/RJ. Os fundamentos teórico-metodológicos são baseados: na concepção de linguagem de Bakhtin, principal categoria de análise dos dados do campo; em Vigotski que forneceu subsídios para um pensamento dialético em torno das culturas infantil e escolar tomadas como textos; em Foucault e Certeau, para analisar as estratégias de poder e das táticas de resistência encontradas nas práticas observadas e suas influências na subjetivação dos sujeitos; na sociologia da infância e no conceito de cultura escolar para contextualizar o campo. Para abordar as transições e as rupturas percebidas nesse processo, as contribuições principais foram definidas a partir de Moss e Corsaro.

Maia (2011) pesquisou duas escolas de educação infantil em um dos cinco municípios mais populosos do Estado do Rio de Janeiro em relação à faixa etária de zero a seis anos, a fim de estudar a infância, a educação infantil e o currículo. Compreende a infância como categoria social e da história, parte da cultura e produtora de cultura, a educação infantil como direito da criança e o currículo como experiência de cultura. Entende ainda que seu objeto de estudo - crianças, professores, gestores
- é um sujeito, ou sujeitos, são sempre sujeitos. A pesquisa indagou porque as escolas de educação infantil organizam seu currículo em torno de datas comemorativas do calendário civil e religioso para compreender porque mesmo com mudanças na educação infantil e com a produção teórica sobre o currículo, essa ainda é a orientação da rede de ensino estudada e das práticas nas escolas de educação infantil e na organização do currículo.

Zlot (2012) estuda as interações entre os sujeitos da escola, os adultos e as crianças. A pesquisa foi realizada em duas turmas de ensino fundamental de uma escola da rede privada do município do Rio de Janeiro. A pesquisa procurou compreender os sujeitos em interação entre si e com o conhecimento. Nessa perspectiva, o estudo questiona como interações entre as crianças interferem na relação com o conhecimento e como também como as interações interferem nos processos de inclusão dos sujeitos no cotidiano da escola. $\mathrm{O}$ estudo das interações também objetivou conhecer/compreender a partir das práticas se espaços e rotinas de colaboração são (ou não) construídos. A pesquisa identificou uma concepção de criança que é ouvida, participa, questiona e colabora, sendo os modos de gestão e as formas de organização do espaço das salas de aula, artefatos e materiais fatores importantes para a construção dessas interações.

Barbosa (2013) faz um estudo comparativo sobre institucionalização e a qualidade das interações na creche. Numa perspectiva etnográfica, foram pesquisadas duas creches, uma pública e outra comunitária, num município da região metropolitana do Rio de Janeiro, acompanhando interações de crianças de três e quatro anos em duas turmas, uma em cada creche. A tese foi o exercício de compreender o cotidiano, levando em conta as crianças como sujeitos que, além de submetidos aos processos de institucionalização e familiarização, também se expressam de forma individual e coletiva, interferindo nesses mesmos processos, mostrando a relação dialética entre as dimensões macro, das políticas, e micro, das interações. As análises revelaram que o mais importante para as crianças nesses dois espaços institucionais são as interações e ações coletivas que, no processo de reprodução interpretativa, favorecem a criação de estratégias para a produção da cultura de pares, tendo a cultura de pares seu locus privilegiado no brincar, mas não se restringindo a ele.

\section{Reflexões teóricas e metodologia: os conceitos em destaque}

As contribuições de Vigotski mais relevantes nas pesquisas do INFOC destacadas no tópico anterior referem-se, primeiramente, à concepção de infância, na qual a criança é vista como sujeito histórico-social, que produz cultura e é produzida na cultura. Nesta perspectiva, as obras com maior referência são os livros $A$ formação social da mente e Imaginação e criação na infância (VIGOTSKI, 2000, 2009). Além disso, a concepção do ser humano como dotado de cultura e história possibilita olhar para as relações como um processo interativo-mediatizado-mediador, de trocas interpessoais que proporcionam a apropriação, produção e reprodução da sociedade. Tal concepção fornece subsídios para um pen- 
samento dialético em torno das culturas infantil e escolar. Em sequência, aparecem as contribuições de Vigotski quanto à concepção de linguagem enquanto espaço das inter-relações sociais e lugar de constituição da consciência, desenvolvimento e formação. Por fim, a relação da criança com o brincar enquanto experiência de cultura e atividade necessária ao seu desenvolvimento.

Os trabalhos também apontam questões de metodologia. Nos textos de Vigotski, ao trazer suas questões para análise, ele está falando também de metodologia. A pesquisa como um processo histórico e cultural, a partir da perspectiva vigotskiana, se constitui numa abordagem dialética que (i) "analisa processos e não objetos" (VIGOTSKI, 2000, p. 81); (ii) estuda o fenômeno do ponto de vista do desenvolvimento, revelando suas relações dinâmicas causais, pois situações externas aparentemente iguais podem ser de naturezas processuais diferentes, mostrando a necessidade de "explicar e não apenas descrever os fenômenos" (VIGOTSKI, 2000, p. 83); (iii) busca a "compreensão da origem e dos processos de estabelecimento do fenômeno". O desafio de buscar a origem e estabelecer como se desenvolve um determinado processo tem o objetivo de "alterar o caráter automático, mecanizado e fossilizado" dos fenômenos (VIGOTSKI, 2000, p. 85).

No próximo tópico, apresentamos um quadro que traz um apanhado mais geral das obras de Vigotski citadas nas teses e dissertações.

\section{Entre grafias e datas: as obras de Vigotski}

As obras citadas aparecem com diferentes grafias e datas de edição, bem como edições em português e espanhol. Há apenas uma citação em inglês (Anexo 2). Para este quadro, consideramos a obra, destacando entre parênteses os diferentes anos de edição.

\section{Considerações finais}

A teoria de Vigotski, ao focar mais nos processos e nas análises e, por isso, privilegiar as relações (KRAMER; SANTOS, 2012), muito contribui para a compreensão das práticas e interações entre crianças e entre estas e adultos, trazendo, em consequência, reflexões para a política de educação infantil e para a formação de professores.

Os modos como os adultos concebem a infância e as crianças interferem nos modos como se relacionam com elas. Pesquisar interações e práticas com crianças entendidas como sujeitos sociais exige metodologias que considerem que "as crianças criam e participam de suas próprias e exclusivas culturas de pares quando selecionam ou se apropriam criativamente de informações do mundo adulto para lidar com suas próprias e exclusivas preocupações" (CORSARO, 2011, p. 31). Exige também "uma relação de alteridade fundamental que emerge de uma diferença de lugar na construção do saber" (AMORIM, 2001, p. 31).

“Ao reconhecer a diferença no 'outro', recuperamos a dignidade de nos reconhecermos nos nossos limites, nas nossas faltas, na nossa incompletude permanente" (PEREIRA; JOBIM e SOUZA, 1998, p. 39). O pesquisador que pretende investigar as crianças como sujeitos deve levar em conta quem são as crianças, como participam do processo de produção dos conhecimentos, que significados produzem sobre a realidade que as cerca (BARBOSA, 2009).

Nesse sentido, a teoria de Vigotski tem contribuído para o estudo sobre a criança, seu desenvolvimento e sua relação com os adultos e com o mundo em um diálogo com a concepção de criança construída pelos teóricos da sociologia da infância.

Quadro 1 - Número de vezes de citação das obras de Vigotski nas teses e dissertações do Infoc de 2003 a 2013

\begin{tabular}{|l|c|}
\hline Obra & $\begin{array}{c}\text { Número de vezes em que } \\
\text { é referenciada }\end{array}$ \\
\hline A construção do pensamento e da linguagem (2001; 2009) & 3 \\
\hline Manuscrito de 1929: psicologia concreta do homem (2000) & 2 \\
\hline A formação social da mente (1991; 1998: 2000; 2003) & 10 \\
\hline Pensamento e Linguagem (1991: 1993: 1998) & 1 \\
\hline Método de investigación In: Obras Escogidas I (1997) & 1 \\
\hline Concrete Human Psychology (1989) & 1 \\
\hline $\begin{array}{l}\text { Psicologia pedagógica: bases psicológicas da aprendizagem e do } \\
\text { desenvolvimento (1991) }\end{array}$ & 1 \\
\hline La imaginacion y el arte em la infância (1987:1996) & 1 \\
\hline Imaginação e criação na infância (2009) & 1 \\
\hline Teoria e método em psicologia (1996) & 1 \\
\hline Obras escogidas II (2001) & 1 \\
\hline Obras escogidas III (1995) & 1 \\
\hline Obras escogidas IV (1996) & 1 \\
\hline Obras escogidas V: fundamentos de defectologia (1997) & 1 \\
\hline O desenvolvimento psicológico na infância (1997) & 1 \\
\hline Psicologia da arte (1998) & 1 \\
\hline A tragédia de Hamlet. o príncipe da Dinamarca (1999) & 2 \\
\hline Psicologia pedagógica (2001) & 1 \\
\hline Linguagem, desenvolvimento e aprendizagem³ (1988) & 1 \\
\hline $\begin{array}{l}\text { Estudos sobre a história do comportamento: o macaco, o primitivo e } \\
\text { a crianca } 4 \text { (1996) }\end{array}$ & \\
\hline Aprendizagem e desenvolvimento intelectual na idade escolar (1998) & \\
\hline Sobre a análise pedológica do processo pedagógico - & \\
\hline
\end{tabular}

\footnotetext{
${ }^{3}$ Em co-autoria com Luria e Leontiev.

${ }^{4} \mathrm{Em}$ co-autoria com Luria.
} 


\section{Referências}

AMORIM, M. O pesquisador e seu outro: Bakhtin nas ciências humanas. São Paulo: Musa, 2001.

BARBOSA, S. N.F. “O que você está escrevendo?"-Na pesquisa com crianças o exercício de compreender e ser compreendido. In: KRAMER, S. Retratos de um desafio: crianças e adultos na educação infantil. São Paulo: Ática, 2009. p. 24-35.

CORSARO, W. Sociologia da infância. Porto Alegre: Artmed, 2011.

KRAMER, S. (Coord.). et al. Relatório da pesquisa: formação de profissionais da educação infantil no estado do rio de janeiro. Rio de Janeiro: Ravil, 2001.

KRAMER, S. (Org.). Profissionais de educação infantil: gestão e formação. São Paulo: Ática, 2005.

KRAMER, S. (Org.). Retratos de um desafio. São Paulo, Ática, 2009.

KRAMER, S.; SANTOS, T. R. L dos. Contribuições de Lev Vigotski para a pesquisa com crianças. In: MARCONDES, M. I.; OLIVEIRA, I. A. de; TEIXEIRA, E. (Org.). Abordagens teóricas e construções metodológicas na pesquisa em educação. Belém, PA: ABEU/UEPA, 2012. p. 17-36.

NUNES, F. F. R.; CORSINO, P.; KRAMER, S. Educação infantil e políticas municipais: em estudo longitudinal. Cadernos de Pesquisa, São Paulo, v. 43, n. 148, p. 152-175, jan./abr. 2013.

PEREIRA, R. M. R.; JOBIM e SOUZA, S. Infância, conhecimento e contemporaneidade. In: KRAMER, S.; LEITE, M. I. (Org.). Infância e produção cultural. Campinas, SP: Papirus, 1998. p. 25-42.

VIGOTSKI, L. S. Formação social da mente. São Paulo: Martins Fontes, 2000.

VIGOTSKI, L. S. Imaginação e criação na infância. São Paulo: Ática, 2009

Recebido em: 29 de agosto de 2013 Aceito em: 05 de novembro de 2014

\section{Anexo 1 - lista completa teses e dissertações defendidas Grupo INFOC (2003/2013)}

\section{Dissertações de Mestrado}

1. BARBOSA, S. N. F. Nas tramas do cotidiano: adultos e crianças construindo a educação infantil. 2004. Dissertação (Mestrado em Educação)-Pontifícia Universidade Católica do Rio de Janeiro, Rio de Janeiro, 2004

2. SIQUEIRA, R. B. A implantação de turmas de educação infantil nas escolas de ensino fundamental: solução ou paliativo? 2011. Dissertação (Mestrado em Educação)Pontifícia Universidade Católica do Rio de Janeiro, Rio de Janeiro, 2011.

3. CASTRO, P. M. O PROINFANTIL no Municipio do Rio de Janeiro: de Agentes Auxiliares de Creche a Professores? 2011. Dissertação (Mestrado em Educação)—Universidade Federal do Rio de Janeiro, Rio de Janeiro, 2011.

4. FONSECA, L. M. Salas de leitura: concepções e práticas. 2004. Dissertação (Mestrado em Educação)-Pontifícia Universidade Católica do Rio de Janeiro, Rio de Janeiro, 2004.
5. MEDEIROS, C. S. Profissionais de Educação, Saúde, Lazer e Cultura que trabalham com a educação infantil: Práticas e Concepções de Infância. 2009. Dissertação (Mestrado em Educação)_Pontifícia Universidade Católica do Rio de Janeiro, Rio de Janeiro, 2009.

6. MELLO, T. F. O. Da mediação do professor às mediações dos sujeitos - adultos e crianças- na educação infantil. 2008. Dissertação (Mestrado em Educação)-Pontifícia Universidade Católica do Rio de Janeiro, Rio de Janeiro, 2008.

7. MENDES, M. F. O diário de uma professora: experiências culturais em alfabetização. 2007. Dissertação (Mestrado em Educação)-Pontifícia Universidade Católica do Rio de Janeiro, Rio de Janeiro, 2007.

8. MOTTA, F. M. As crianças e as práticas de autoridade: um estudo na educação infantil. 2007. Dissertação (Mestrado em Educação)—Pontifícia Universidade Católica do Rio de Janeiro, Rio de Janeiro, 2007.

9. MOURA, M. T. J. A. Arte e Infância: um estudo das interações entre crianças, adultos e obras de arte em museu. 2005. Dissertação (Mestrado em Educação)Pontifícia Universidade Católica do Rio de Janeiro, Rio de Janeiro, 2005.

10. NASCIMENTO, A. Infância e cidade: crianças e adultos em uma pracinha do Rio de Janeiro. 2004. Dissertação (Mestrado em Educação)-Pontifícia Universidade Católica do Rio de Janeiro, Rio de Janeiro, 2004.

11. SANTOS, L. M. Alfabetização e Letramento: fios que tecem a leitura e a escrita no cotidiano da escola Beta. 2004. Dissertação (Mestrado em Educação)_Pontifícia Universidade Católica do Rio de Janeiro, Rio de Janeiro, 2004

12. SANTOS, N. O. Infância, práticas culturais e consumo: um olhar sobre crianças e adultos numa escola pública. 2005. Dissertação (Mestrado em Educação)-Pontifícia Universidade Católica do Rio de Janeiro, Rio de Janeiro, 2005.

13. SCRAMINGNON, G. "Eu lamento, mas é isto que nós temos": o lugar da creche e de seus profissionais no município do Rio de Janeiro. 2011. Dissertação (Mestrado em Educação)Universidade Federal do Estado do Rio de Janeiro - UNIRIO, Rio de Janeiro, 2011.

14. PIO BORGES, L. T. Relações e concepções de crianças com/sobre a natureza: um estudo em uma escola municipal. 2010. Dissertação (Mestrado em Educação) Pontifícia Universidade Católica do Rio de Janeiro, Rio de Janeiro, 2010.

15. MAIA, M. N. educação infantil: com quantas datas se faz um currículo? 2011. Dissertação (Mestrado em Educação)—Pontifícia Universidade Católica do Rio de Janeiro, Rio de Janeiro, 2011

16. BARROS, C. O. "Ainda temos muito para caminhar, mas a menina dos olhos é a formação": políticas públicas e escolaridade de profissionais da educação infantil. 2012. Dissertação (Mestrado) —Pontifícia Universidade Católica do Rio de Janeiro, Rio de Janeiro, 2012.

17. ZLOT, G. R. W. Crianças, adultos e interações na escola: encontros no cotidiano. 2012. Dissertação (Mestrado em Educação)—Pontifícia Universidade Católica do Rio de Janeiro, Rio de Janeiro, 2012

18. RONCARATI, M. Perspectivas de uma educação dialógica na creche: a co-autoria da criança na construção 
da prática educativa. 2012. Dissertação (Mestrado em Educação)Universidade Federal do Estado do Rio de Janeiro - UNIRIO, Rio de Janeiro, 2012.

Teses de Doutorado:

1. BARBOSA, S. N. F. Vem, agora eu te espero institucionalização e qualidade das interações na creche: um estudo comparativo. 2013. Tese (Doutorado) Departamento de Educação, Pontifícia Universidade Católica do Rio de Janeiro, Rio de Janeiro, 2013.

2. CORSINO, P. Infância, linguagem e letramento: educação infantil na rede municipal de ensino do Rio de Janeiro. 2003. Tese (Doutorado em Educação) - Pontifícia Universidade Católica do Rio de Janeiro, Rio de Janeiro, 2003.

3. DEBORTOLI, J. A. O. Infâncias na creche: corpo e memória nas práticas e nos discursos da educação infantil: um estudo de caso em Belo Horizonte. 2004. Tese (Doutorado em Educação)—Pontifícia Universidade Católica do Rio de Janeiro, Rio de Janeiro, 2004.

4. DRAGO, R. Infância, educação infantil e inclusão: um estudo de caso em Vitória. 2005. Tese (Doutorado em Educação)—Pontifícia Universidade Católica do Rio de Janeiro, Rio de Janeiro, 2005.

5. FISCHBERG, J. Leitura de Jovens. 2011. Tese (Doutorado em Educação)-Pontifícia Universidade Católica do Rio de Janeiro, Rio de Janeiro, 2011.

6. GUIMARÃES, D. O. Relações sociais, linguagem $e$ documentação pedagógica reconstruindo as ações com as crianças pequenas. 2007. Tese (Doutorado em Educação)—Pontifícia Universidade Católica do Rio de Janeiro, Rio de Janeiro, 2007.

7. LIMA, M. B. Práticas cotidianas e identidades étnicas: um estudo no contexto escolar. 2006. Tese (Doutorado em Educação)-Pontifícia Universidade Católica do Rio de Janeiro, Rio de Janeiro, 2006.

8. MICARELLO, H. A. L. S, Professores da pré-escola: trabalho, saberes e processos de construção de identidade. 2006. Tese (Doutorado em Educação)—Pontifícia Universidade Católica do Rio de Janeiro, Rio de Janeiro, 2006.

9. MOTTA, F. De crianças a alunos: transformações sociais na passagem da educação infantil para o ensino fundamental. 2010. Tese (Doutorado)Departamento de Educação, Pontifícia Universidade Católica do Rio de Janeiro, Rio de Janeiro, 2010.

10. NUNES, M. F R. educação infantil no Estado do Rio de Janeiro: um estudo das estratégias municipais de atendimento. 2005. Tese (Doutorado em Educação)Universidade Federal do Rio de Janeiro, Rio de Janeiro, 2005.

11. TIRIBA, L. Crianças, natureza e educação infantil. 2005. Tese (Doutorado em Educação) —Pontifícia Universidade Católica do Rio de Janeiro, Rio de Janeiro, 2005.

12. FREIRE, E. F. Pelas telas de um aramado: educação infantil, cultura e cidade. 2008. Tese (Doutorado em Educação)-Pontifícia Universidade Católica do Rio de Janeiro, Rio de Janeiro, 2008.

\section{Anexo 2 - obras referenciadas nas teses e dissertações:}

VIGOTSKI, L. S. La Imaginacion y el arte em la infância. México: Hispanicas, 1987.

VIGOTSKI, L. S. "Concrete Human Psychology". Soviet Psychology, [S.1.], v. 27, n. 2, p. 53-77, 1989.

VYGOTSKY, L. S. A formação social da mente. São Paulo: Martins Fontes, 1991.

VIGOTSKI, L.S. La imaginacion y el arte em la infância. Madrid: Akhal, 1996.

VIGOTSKI, L. S. Método de investigación. In: Obras Escogidas. Madrid: Visor, 1997. v. 1, p. 47-95.

VIGOTSKI, L. S. Pensamento e Linguagem. São Paulo: Martins Fontes, 1998.

VIGOTSKI, L. S. A formação social da mente. 6. ed. São Paulo: Martins Fontes, 2000.

VIGOTSKI, L. S. Manuscrito de 1929. Educação \& Sociedade, Campinas, v. 21, n. 71, p. 21-44, jul. 2000.

VIGOTSKI, L. S. A construção do pensamento e da linguagem. Tradução de Paulo Bezerra. São Paulo: Martins Fontes, 2001.

VIGOTSKI, L. S. A formação social da mente. São Paulo: Martins Fontes, 2003.

VIGOTSKI, L. S. A construção do pensamento e da linguagem. São Paulo: Martins Fontes, 2009.

VIGOTSKI, L. S Imaginação e criação na infância. São Paulo: Ática, 2009.

VIGOTSKI, L. S. Sobre a análise pedológica do processo pedagógico. In: PRESTES, Z. R. Quando não é quase a mesma coisa: análise de traduções de Lev Semionovitch Vigotski no Brasil. Repercussões no campo educacional. 2010. Tese (Doutorado em Educação)Universidade de Brasília, Brasília, 2010. p. 261-281.

VYGOTSKY, L. S.; LURIA, A. R.; LEONTIEV, A. Linguagem, desenvolvimento e aprendizagem. São Paulo: Ícone/EDUSP, 1988.

VYGotsky, L. S. Pensamento e linguagem. São Paulo: Martins Fontes, 1991.

VYGOTSKY, L. S. Psicologia pedagógica: bases psicológicas da aprendizagem e do desenvolvimento. São Paulo: Moraes, 1991.

VYGOTSKY, L. S. Pensamento e linguagem. São Paulo: Martins Fontes, 1993.

VYGOTSKY, L.S. Obras escogidas. Madrid, Visor, 1995. v. 3. VYGOTSKY, L.S. Obras escogidas. Madrid, Visor, 1996. v. 4.

VYGOTSKY, L. S. Teoria e método em psicologia. São Paulo: Martins Fontes, 1996

VYGOTSKY, L. S.; LURIA, A. R. Estudos sobre a história do comportamento: o macaco, o primitivo e a criança. Porto Alegre: Artes Médicas, 1996.

VYGOTSKY, L.S. Obras escogidas: fundamentos de defectologia. Madrid, Visor, 1997. v. 5.

VYGOTSKY, L. S. O desenvolvimento psicológico na infância. São Paulo: Martins Fontes, 1997.

VYGOTSKY, L. S. A formação social da mente. 6. ed. São Paulo: Martins Fontes, 1998. 
VYGOTSKY, L. S. Aprendizagem e desenvolvimento intelectual na idade escolar. In: VYGOTSKY. L. S.; LURIA, A. R.; LEONTIEV, A. Linguagem, desenvolvimento $e$ aprendizagem. São Paulo: Ícone/USP, 1998. p.103-118.

VYGOTSKY, L. S. Psicologia da arte. São Paulo: Martins Fontes, 1998.

VYGOTSKY, L. S. A tragédia de Hamlet, o príncipe da Dinamarca. São Paulo: Martins Fontes, 1999.

VYGOTSKY, L. S. Psicologia pedagógica. São Paulo: Martins Fontes, 2001

VYGOTSKY, L.S. Obras escogidas. Madrid, Visor, 2001. v. 2. 